\title{
Indian Solutions for Indian Problems-Association of Breast Surgeons of India (ABSI) Practical Consensus Statement, Recommendations, and Guidelines for the Treatment of Breast Cancer in India
}

\author{
S. P. Somashekhar ${ }^{1} \cdot$ Gaurav Agarwal $^{2}$ - S. V. S. Deo ${ }^{3} \cdot$ Chintamani $^{4} \cdot$ P. Raghu Ram ${ }^{5}$. \\ Diptendra Sarkar ${ }^{6}$ - Vani Parmar ${ }^{7}$
}

Received: 13 June 2017 / Accepted: 13 June 2017 / Published online: 22 June 2017

(C) Association of Surgeons of India 2017

\section{Introduction}

Breast cancer is considered as the most common type of female cancer accountable for nearly $23 \%$ of all cancers in women globally $[1,2]$. It is believed that global prevalence of breast cancer will cross two million by the year 2030 which will have the major contributors from developing countries [3]. As far as India is concerned, the incidence rates vary across the country with northeast and metropolitan cities (Mumbai, New Delhi) showing the highest rates [4]. The factors which contribute to this variation include demographic differences (education), lifestyle factors (use of tobacco and alcohol), anthropometric factors (adiposity), and reproductive factors (age at first child and number of children) [4]. One of the most common contributors to the high mortality rate is due to the diagnosis at advance stages of the disease which can be due to low awareness, incomplete treatment regimens, and limited access to effective treatment at regional cancer centers [5-10]. As per National Breast Cancer Foundation, currently available treatments for breast cancer are based on a number

S. P. Somashekhar

somusp@yahoo.com

Manipal Comprehensive Cancer Center, Bengaluru 560017, India

2 SGPGI, Lucknow, India

3 AIIMS, New Delhi, India

4 Vardhaman Mahavir Medical College, Safdarjung Hospital, New Delhi, India

KIMS, Hyderabad, India

6 IPGIMER, Kolkata, India

7 Tata Memorial Hospital, Mumbai, India of factors including size of tumor in relation to size of breast, result of specific pathology tests (hormone receptors, HER2 receptors), menopause, age, general health conditions, family history, or other risk factors [11]. The treatments include surgery, chemotherapy, radiation therapy, hormone therapy, and targeted therapy along with the involvement of nutrition and physical activity and follow-up care.

According to a report published in Breast Cancer Deadline 2020 , the death rate is gradually declining after 1990 which attributes to early detection, awareness, and continuous improvement in treatment, which is a good sign [12]. Although the advancement of new technology provides hope to cure of breast cancer, still there are barriers to provide an optimal treatment to the patients globally, especially in the developing countries. So it's very important to have a universal approach and guidelines at place, especially in India where the lack of awareness among the patients is quite high. The present manuscript is developed with the help of key opinion leaders (KOLs)/experts in domain, published evidences, and practical experiences in real-life management of breast cancer to draft the consensus guidelines towards finding Indian solutions for Indian problems.

\section{Background and Methods}

St. Gallen Consensus Conference on early breast cancer is conducted which provided mostly evidence-based, globally valid treatment recommendations for breast cancer care, with a broad spectrum of acceptable clinical practice [13]. In the year 2015, Vienna, Austria conducted St. Gallen Consensus Conference on early breast cancer, and some recommendations on the current and newer practices were added to the official St. Gallen Consensus publication [14]. These are the 
globally accepted recommendations for the management of breast cancer.

In developing countries like India, there is a need to develop the guidelines to streamline the management of breast cancer. To address this issue, the expert panel of KOLs met to discuss and arrive at a consensus statement to provide community oncologists practical guidelines on the management of breast cancer in Indian patients. The discussions were based on the current practices and scenario which exist in India. The mandate was to develop practical consensus recommendations applicable globally with emphasis on countries with limited resources. The core expert group discussed over several sessions and arrived at a consensus on the methodology to be used as well as develop the survey questionnaire.

The questionnaire was developed by a panel of experts from academia and ABSI, and these were in sync with the St. Gallen Consensus panel questions which suited to Indian needs and included limitations to evaluation, surgical treatment, surgical pathology, and adjuvant/neo-adjuvant treatment. The questions were provided with three to five options - numbered 1 to 5; one of which is most appropriate answer and also had abstain option. The series of multiple choice questions included key practical issues and management challenges. Each question was projected to panel of experts and audiences and voting was comprised by experts and audiences simultaneously during the fourth ABSICON held at Bangalore from 1 to 3 July 2016. Thus, at the end of every question, audiences' views and KOLs' views were collected. The survey answers were used as the basis for formulating the consensus statement, so that community oncologists have a ready-to-use practical consensus recommendation for breast cancer management. The ABSI 2016 will therefore serve to optimize management of breast cancer in conjunction with evolving literature, good clinical judgment, and individual patient characteristics and preferences.

A total of 16 broad question categories containing 47 unique questions were part of the expert group discussions (Table 1).

\section{Highlights of the ABSI Consensus Statement on Breast Cancer}

\section{Breast Cancer Evaluation}

There were three questions related to the evaluation of breast cancer and the answers were as follows:

A. Core biopsy is mandatory/preferred modality for diagnosis of breast cancer: $90.9 \%$ of audiences had affirmative opinion in comparison to $95.2 \%$ of experts.

B. Metastatic work-up: Should be done for stage I and II breast cancers $(4.7 \%$ (audience) vs $0 \%$ (expert)), recommended for stage III breast cancer $(55.7 \%$ (audience) vs $75 \%$ (expert)), and should be done in all breast cancer patients (39.6\% (audience) vs $25 \%$ (expert)).

C. Positron emission tomography (PET) scan is preferred modality in clinically isolated recurrences or ambiguous lesion on conventional imaging and NOT for routinely staging all breast cancer patients: $87.6 \%$ of audiences had affirmative opinion in comparison to $76.2 \%$ of experts.

Expert Group Consensus Early breast cancer may be asymptomatic and there may be absence of pain and discomfort. So, it is very important to have a proper evaluation of the breast cancer. For the evaluation, the experts recommended mandatory core biopsy (Fig. 1), metastatic work-up for stage III breast cancer, and an early diagnosis which may enable an oncologist to provide an ideal treatment for management of breast cancer. This triple assessment is mandatory for breast cancer evaluation.

\section{Breast Cancer Screening in India}

The panel drafted one question related to breast cancer screening in India; question and the result were as follows:

The most applicable/ pragmatic screening modality for breast cancer in Indian scenario: mammography (20.2\% (audience) vs $05 \%$ (expert)), clinical breast examination (55.0\% (audience) vs $60 \%$ (expert)), and breast selfexamination (28.8\% (audience) vs $20 \%$ (expert)).

Expert Group Consensus Low cancer awareness, presence of stigma, fear, gender inequity, and decreased involvement in screening behavior (e.g., breast cancer self-examination) attribute to high mortality rates among women in India [8]. The audiences and experts have comparable responses, and the experts recommended clinical breast examination (Fig. 2) to screen the cancer which can be possible through proper awareness among women in India.

\section{Surgery of the Primary (Early Breast Cancer (EBC))}

This was one of the most important categories, and there were six questions drafted by the panel for the audiences and experts. The questions and the opinions were as follows:

A. For the women undergoing breast conserving surgery for invasive EBC and proceeding to standard radiation and adjuvant systemic therapy the minimum acceptable surgical margin: no ink on invasive tumor $(37.5 \%$ (audience) vs $72.7 \%$ (expert)), 1-2-mm clearance ( $26.9 \%$ (audience) vs $9.1 \%$ (expert)), $>2-5$-mm clearance ( $12.5 \%$ (audience) vs $9.1 \%$ (expert)), and $>5$-mm clearance $(22.1 \%$ (audience) vs $4.5 \%$ (expert)). 
Table 1 Question categories addressed by the breast cancer expert group

\begin{tabular}{lll}
\hline S. no. & Broad question category: breast cancer & Number of questions \\
\hline 1 & Breast cancer evaluation & 03 \\
2 & Breast cancer screening in India & 01 \\
3 & Surgery of primary EBC & 06 \\
4 & Management of locally advanced breast cancer & 04 \\
5 & Surgical management of LABC & 03 \\
6 & Management of Axilla in EBC & 07 \\
7 & Management of Axilla in LABC/post-NACT patients & 01 \\
8 & Oncoplastic surgical principles & 02 \\
9 & Post-mastectomy reconstruction & 01 \\
10 & Risk reducing surgery & 01 \\
11 & Adjuvant radiotherapy & 07 \\
12 & Surgical pathology & 01 \\
13 & Biomarkers & 01 \\
14 & Molecular profiling of breast cancer & 02 \\
15 & Adjuvant hormonal therapy & 05 \\
16 & Breast cancer in young patients & 02 \\
Total & & 47 \\
\hline
\end{tabular}

$E B C$ early breast cancer, $L A B C$ locally advanced breast cancer, $N A C T$ neo-adjuvant chemotherapy
B. Unilateral multifocal and multicentric tumors can be treated with en-bloc excision, provided margins are clear, good cosmesis is ensured, and whole breast RT is planned: Yes (42.5\% (audience) vs $85.7 \%$ (expert)) and never $(52.8 \%$ (audience) vs $9.5 \%$ (expert)).

C. Should the width of margin that needs to be excised dependent on tumor biology (grade, hormone receptor, HER2neu)?: Yes (17.6\% (audience) vs $05.0 \%$ (expert)) and no $(77.8 \%$ (audience) vs $95 \%$ (expert)).

D. Should the width of margin that needs to be excised be greater after neo-adjuvant therapy?:Yes $(18.8 \%$ (audience) vs $14.3 \%$ (expert)) and no ( $80.2 \%$ (audience) vs $81 \%$ (expert)).
E. Frozen section for margin assessment during breast conservation surgery should be: done in all classes $(29.7 \%$ (audience) vs $10.0 \%$ (expert)), in select patients where indicated, it is preferable if expertise and infrastructure exists (62.4\% (audience) vs $60.0 \%$ (expert)), and not recommended ( $7.9 \%$ (audience) vs $30 \%$ (expert)).

F. In case margin(s) are found positive after breast conservation surgery, which statement is most appropriate: Reexcision is not mandatory if up to two margins are found infiltrated (14.2\% (audience) vs $9.5 \%$ (expert)), and reexcision is mandatory in all patients, except if skin/deep fascial margin only is positive ( $76.4 \%$ (audience) vs $85.7 \%$ (expert)).
Fig. 1 Opinion on core biopsy (audiences vs experts)

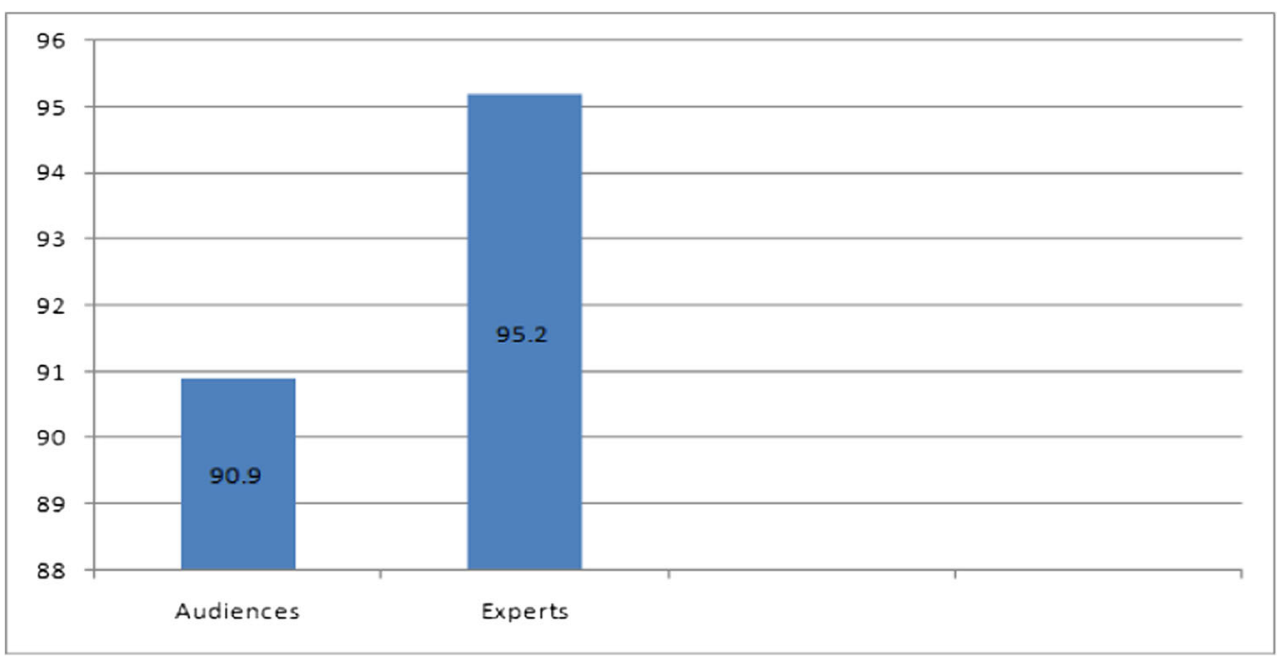


Fig. 2 Breast cancer screening in India

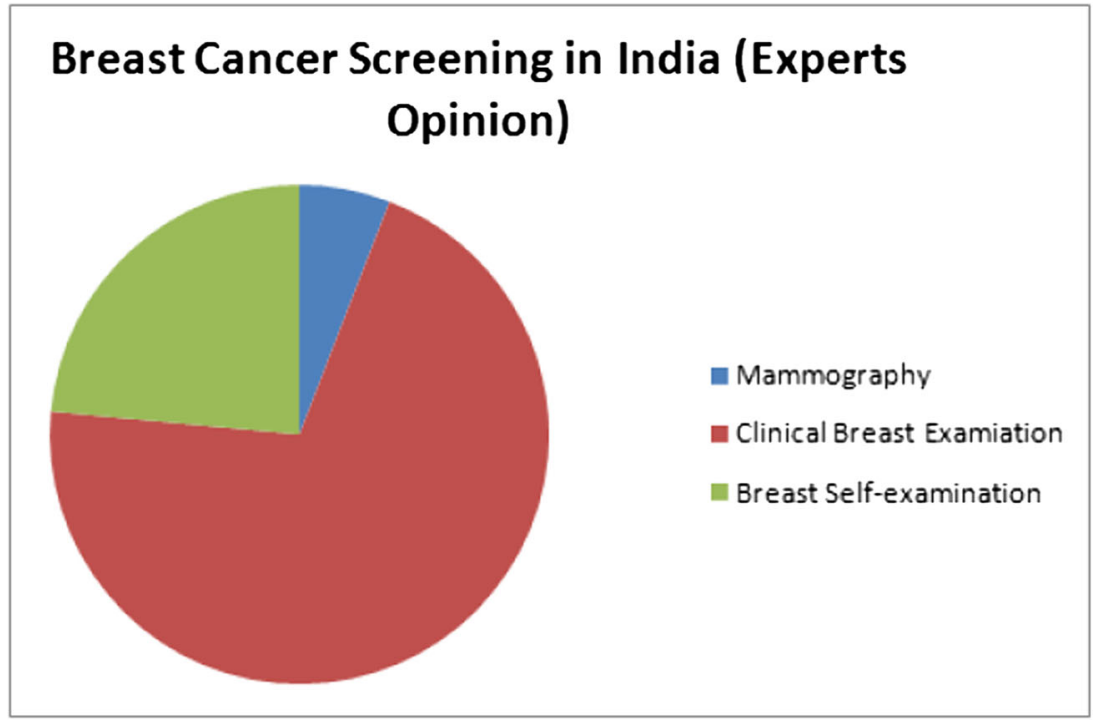

Expert Group Consensus For the surgical treatment of EBC, the experts recommended no ink on invasive tumor as the minimum acceptable surgical margin, en-block excision for treatment of unilateral multifocal and multicentric tumors. The experts also strongly recommended that width of margin that needs to be excised is independent of tumor biology, and it should not be greater after neo-adjuvant therapy. During surgery, frozen section for margin assessment should be preferred if expertise and infrastructure exist; and for the situations where case margin(s) are found positive after breast conservation surgery, the experts recommended mandatory reexcision in all patients, except if skin/deep fascial margin only is positive. The researches indicated that oncoplastic surgery results in excision of larger volume of breast tissue and correspondingly obtains wider surgical margins as compared to conventional breast conservation surgery [15].

\section{Management of Locally Advanced Breast Cancer (LABC)}

The panel drafted four questions for this category. The questions and the response of audiences and experts are provided below:

A. Neo-adjuvant chemotherapy is the standard of care in majority of locally advanced breast cancer patients: Yes (92\% (audience) vs $90.5 \%$ (expert0) and no $(6.6 \%$ (audience) vs $9.5 \%$ (expert)).

B. Most effective and practical regime for neo-adjuvant chemotherapy (NACT) includes anthracycline alone (11.7\% (audience) vs $5.9 \%$ (expert)), taxanes alone $(9.7 \%$ (audience) vs $0 \%$ (expert)), anthracycline followed by taxanes $(69.7 \%$ (audience) vs $82.4 \%$ (expert)).

C. Best sequence/schedule of treatment for LABC patient is complete whole of NACT followed by surgery and RT
(31.3\% (audience) vs $66.7 \%$ (expert)), complete part of NACT followed by surgery, rest of chemo, and RT (66\% (audience) vs $33.3 \%$ (expert)).

D. Best time to assess histology and hormone receptors in patients treated with NACT: Before initiation of NACT ( $94.5 \%$ (audience) vs $94.4 \%$ (expert)), after 2 cycles of NACT ( $1.4 \%$ (audience) vs $0 \%$ (expert)), after completion of NACT at surgery (2.7\% (audience) vs $0 \%$ (expert)).

Expert Group Consensus For the management of LABC, the experts strongly batted for the neo-adjuvant chemotherapy as the standard of care for most of the LABC patients and anthracycline followed by taxanes as preferred and most effective practical regime for NACT. As per the experts, the sequence or schedule of treatment for LABC patients should be NACT followed by surgery and RT, and histology and hormone receptors in patients treated with NACT should be evaluated before initiation of NACT. These are the recommendations based on practical scenarios in India and can be differed as per actual/specific situations. Advances in systemic therapy, including radiation treatment, surgical management, and the development of new targeted agents, have significantly improved clinical outcomes for patients with LABC which can be used to provide optimum solutions for Indian patients [16].

\section{Surgical Management of LABC}

The panel drafted three questions for this category. The questions and the response of audiences and experts are provided below:

A. In LABC patients undergoing post-neo-adjuvant chemotherapy breast conservation surgery, which statement is 
most appropriate: All LABC patients are potential candidates for post-NACT-breast cancer surgery (BCS) ( $16.2 \%$ (audience) vs $26.3 \%$ (expert)), only a subset of LABC can be offered NACT BCS (74.3\% (audience) vs $73.7 \%$ (expert)), BCS should not be offered to LABC patients $(8.1 \%$ (audience) vs $0 \%$ (expert)).

B. Patients planned for NACT followed by BCS should have periphery of tumor marked before initiation or after first cycle of NACT (40.7\% (audience) vs $35.3 \%$ (expert)), core of the tumor marked before initiation or after first cycle of NACT (48.3\% (audience) vs $58.8 \%$ (expert)), and marking the tumor site not needed $(9.7 \%$ (audience) vs $0 \%$ (expert)).

C. In patient whose tumor has responded to NACT, at surgery the extent of tissue to be excised at BCS: wide of original (pre-NACT) margins (36.6\% (audience) vs $12.5 \%$ (expert)), wide of current (post-NACT) margins $(60.0 \%$ (audience) vs $87.5 \%$ (expert)), and whole quadrant of breast (3.4\% (audience) vs $0 \%$ (expert)).

Expert Group Consensus For the surgical management of LABC, the experts had the opinion that only a subset of LABC can be offered NACT BCS depending on the specifications, and the patients planned for NACT followed by BCS should have core of the tumor marked before initiation or after first cycle of NACT; although the patients could also have periphery of tumor marked before initiation or after first cycle of NACT for specific situations, if applicable. For the patients who have a positive tumor response to NACT, during surgery the extent to tissue to be excised at BCS should be wide of current (post-NACT) margins.

\section{Management of Axilla in EBC}

This category was very important and the panel drafted seven questions for this category. The questions and the response of audiences and experts are provided below:

A. USG-guided FNAC is preferable for preoperative staging of axilla before SLNB/ALND: Yes (45.2\% (audience) vs $72.2 \%$ (expert)) and no $(53.4 \%$ (audience) vs $22.2 \%$ (expert)).

B. Sentinel lymph node biopsy should be considered in clinically: Node negative axilla (93.8\% (audience) vs $93.3 \%$ (expert)) and node positive axilla $(6.2 \%$ (audience) vs $6.7 \%$ (expert)).

C. For sentinel lymph node biopsy (SLNB) technique which is the most appropriate statement: SLNB using blue dye alone can be performed in resource constrained setup (86.3\% (audience) vs $63.2 \%$ (expert)) and combination of radioisotope and blue dye is mandatory for SLNB (12.3\% (audience) vs $21.1 \%$ (expert)).

D. What is the acceptable false negative rate for SLNB: Up to $5 \%$ (43.8\% (audience) vs $47.1 \%$ (expert)), up to $10 \%$ ( $46.6 \%$ (audience) vs $47.1 \%$ (expert)), and up to $20 \%$ ( $2.7 \%$ (audience) vs $0 \%$ (expert)).

E. Molecular analysis should be routinely performed for SLN assessment: Yes (13.1\% (audience) vs 10.0\% (expert)) and no ( $83.4 \%$ (audience) vs $90.0 \%$ (expert)).

F. Is completion ALND mandatory after micro-metastasis is identified in SLN: Yes (40.7\% (audience) vs 5.6\% (expert)) and no (55.9\% (audience) vs $88.9 \%$ (expert)).

G. Should completion ALND be considered mandatory if macro-metastasis is identified in SLN: Yes $(92.4 \%$ (audience) vs $61.1 \%$ (expert)) and no (6.9\% (audience) vs $33.3 \%$ (expert)).

Expert Group Consensus For the management of axilla in EBC, the experts strongly recommended USG-guided FNAC for preoperative staging of axilla before SLNB/ALND and SLNB should be considered in clinically node negative axilla which can be performed in resource constrained setup using blue dye in countries like India; the acceptable false negative rate for SLNB can vary from 5 to $10 \%$. For SLN assessment, routine molecular analysis was not recommended, and completion ALND is not mandatory after identification of micrometastasis in SLN but it should be mandatory if macrometastasis is identified in SLN. In current Indian scenario, the SLNB is now increasingly being considered the favored method to treat low-volume axilla, and various studies have proved its significance [17].

\section{Management of Axilla in LABC/ Post-NACT Patients}

This category had one question given below:

Which is the most appropriate statement for managing axilla in a patient planned to be treated with neoadjuvant chemotherapy: SLNB should be performed before NACT (26.1\% (audience) vs $26.7 \%$ (expert)), SLNB should be performed after NACT $(34.5 \%$ (audience) vs $66.7 \%$ (expert)), SLNB is not recommended in postNACT patients $(26.1 \%$ (audience) vs $0 \%$ (expert)), and ALND can be avoided if one SLN is metastatic (7\% (audience) vs $0 \%$ (expert)).

Expert Group Consensus For the management of axilla in LABC/post-NACT patients, experts recommended that SLNB should be performed after NACT for the patients planned to be treated with NACT. 


\section{Oncoplastic Surgical Principles that Every Breast Surgeon Should Know}

The panel drafted two questions for this category. The questions and the response of audiences and experts are provided below:

A. After how much volume loss oncoplastic procedure should be considered after BCS: $10 \%$ (3.5\% (audience) vs $14.3 \%$ (expert)), $20 \%$ (77.1\% (audience) vs $71.4 \%$ (expert)), and $30 \%$ (16.0\% (audience) vs $7.1 \%$ (expert)).

B. Central tumors are contraindications to breast conservation surgery: Yes (18.6\% (audience) vs 6.7\% (expert)) and no $(81.4 \%$ (audience) vs $86.7 \%$ (expert)).

Expert Group Consensus The experts recommended that after BCS, if the volume loss is $20 \%$ then oncoplastic procedure should be considered and central tumors are not contraindications to breast conservation surgery. These are two oncoplastic surgical principles that every breast surgeon should know.

\section{Post-mastectomy Reconstruction}

This category had one question given below:

Preferred timing of post-mastectomy breast reconstruction: Immediate $(76.2 \%$ (audience) vs $77.8 \%$ (expert)) and delayed (20.3\% (audience) vs $22.2 \%$ (expert)).

Expert Group Consensus The experts strongly recommended that post-mastectomy breast reconstruction should be immediately performed (Fig. 3). As per the researches, breast reconstruction after resection of breast cancers is helpful in increasing the psychosocial functioning and quality of life among the treated patients [18].

\section{Risk-reducing Surgery}

This category had one question given below:

Which of the following procedures can be recommended for risk reduction in BRCA-positive women: Prophylactic bilateral mastectomy (29.3\% (audience) vs $5.9 \%$ (expert)), prophylactic bilateral salpingo-oophorectomy (10.0\% (audience) vs $5.9 \%$ (expert)), and both $(54.3 \%$ (audience) vs $88.2 \%$ (expert)).

Expert Group Consensus The experts recommended that prophylactic bilateral mastectomy and prophylactic bilateral salpingo-oophorectomy can be performed for risk reduction in BRCA-positive women (Fig. 4). However, studies have suggested that total mastectomy provides the greatest breast cancer risk reduction due to removal of more breast tissue [19]. These surgeries are highly effective as bilateral prophylactic mastectomy has been shown to reduce the risk of breast cancer by at least $95 \%$ in women who have a deleterious mutation in the BRCA1 gene or the BRCA2 gene [20-23], while bilateral prophylactic salpingo-oophorectomy has been shown to reduce the risk of breast cancer by approximately $50 \%$ in women who have high risk of developing the disease [19].

\section{Adjuvant RT}

The panel drafted seven questions for this category. The questions and the response of audiences and experts are provided below:

A. Is post-mastectomy radiotherapy recommended in T1/T2 with 1-3 node positive patients: Yes, without exception (25.9\% (audience) vs $6.2 \%$ (expert)), yes, but can be omitted in selected patients $(52.5 \%$ (audience) vs $62.5 \%$ (expert)), and no (20.9\% (audience) vs $31.2 \%$ (expert)).

B. Does axillary radiation has any therapeutic role after complete ALND: Yes (33.3\% (audience) vs 31.2\% (expert)) and no $(63.8 \%$ (audience) vs $68.8 \%$ (expert)).

C. Tumor bed radiation boost is mandatory following BCS for invasive ductal cancer: Yes $(90.8 \%$ (audience) vs $64.7 \%$ (expert)) and no (7\% (audience) vs $35.3 \%$ (expert)).

D. Most appropriate statement regarding accelerated partial breast radiation (APBI): APBI should be offered to a select group of early breast cancer patients $(68.6 \%$ (audience) vs 93.3\% (expert)) and APBI should be offered to all patients undergoing BCS (23.6\% (audience) vs $0 \%$ (expert)).

E. In patients with T1 tumor and 1-2 metastatic SLNs, radiotherapy to axilla is a valid option instead of surgery in select cases?: Yes (59.2\% (audience) vs $88.9 \%$ (expert)) and no (38.7\% (audience) vs 5.6\% (expert)).

F. Can radiotherapy be omitted in elderly and low-risk patients undergoing BCS: Yes $(66.2 \%$ (audience) vs $87.5 \%$ (expert)) and no (29.7\% (audience) vs $12.5 \%$ (expert)).

G. Approach to RT after neo-adjuvant therapy is based on: the stage before neo-adjuvant therapy (64.3\% (audience) vs $76.5 \%$ (expert)), stage after neo-adjuvant therapy (32.9\% (audience) vs $23.5 \%$ (expert)).

Expert Group Consensus The experts recommended that radiotherapy is recommended in T1/T2 with 1-3 node positive patients but can be omitted in selected patients and axillary radiation has no therapeutic role after complete 
Fig. 3 Preferred timing of postmastectomy breast reconstruction

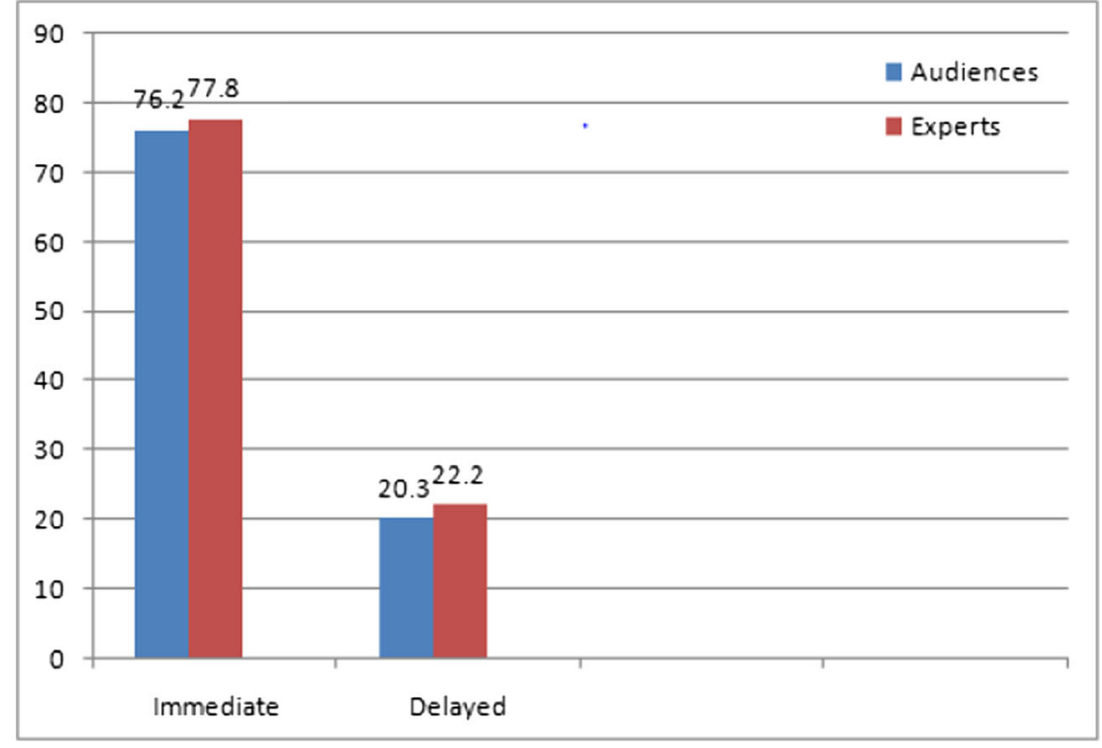

ALND. Experts batted for tumor bed radiation boost following BCS for invasive ductal cancer based on selected patients. Experts strongly recommended that APBI should be offered to a select group of early breast cancer patients and for patients with T1 tumor and 1-2 metastatic SLNs, radiotherapy to axilla is a valid option instead of surgery in select cases. The experts advised to omit the radiotherapy for elderly and low-risk patients undergoing BCS and suggested that cancer stage before the neo-adjuvant therapy forms the base for radiotherapy.

After mastectomy, adjuvant treatment may include local irradiation, systemic therapy with cytotoxic chemotherapy, or endocrine therapy. The adjuvant treatment was considered useful, and a decrease was recorded in breast cancer mortality in the US and UK [24].

\section{Surgical Pathology}

This category had one question given below:

Standardized reporting of breast histology including HP type, grade, margins, tumor numbers and size, lymph nodes, numbers, sizes, and number of metastatic nodes is desirable in most cases (11.6\% (audience) vs $0 \%$ (expert)) and mandatory in ALL patients (87.7\% (audience) vs $100 \%$ (expert)).

Expert Group Consensus The experts strongly recommended that reporting of breast histology must be mandatory in practice which should include HP type, grade, margins, tumor
Fig. 4 Expert opinion for riskreducing surgery

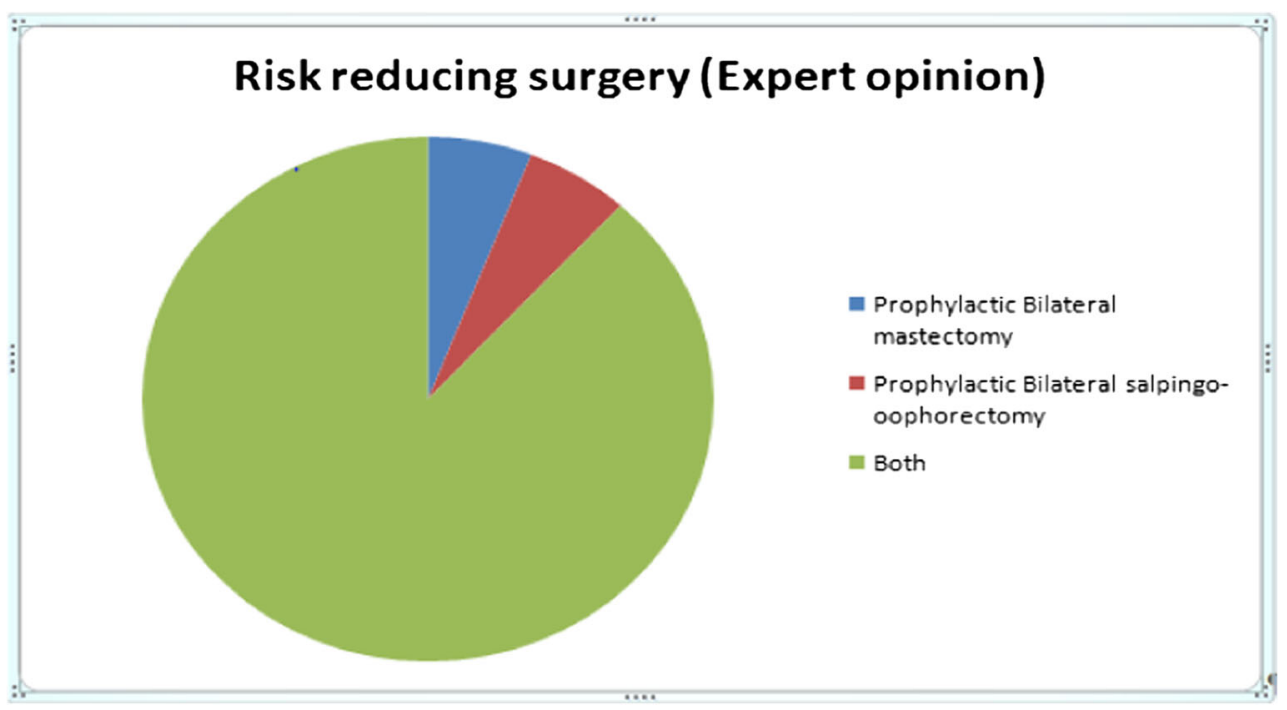


numbers and size, lymph nodes, numbers, sizes, and number of metastatic nodes.

\section{Biomarkers}

This category had one question given below:

Minimum biomarkers that should be tested in breast pathology: IHC for ER and PR (7.5\% (audience) vs $0 \%$ (expert)), IHC for ER, PR and HER2neu (65.8\% (audience) vs $64.7 \%$ (expert)), IHC for ER, PR, and FISH for HER2neu (8.9\% (audience) vs 0\% (expert)), and ER, PR, HER2neu, and Ki67 (17.1\% (audience) vs $35.3 \%$ (expert)).

Expert Group Consensus The experts recommended that IHC for ER, PR, and HER2neu should be tested in breast pathology as a minimum biomarker. For selected patients, IHC for ER, PR, HER2neu, and Ki67 may also be tested.

\section{Molecular Profiling of Breast Cancer}

This category had two questions given below:

A. Multigene signature testing is routinely recommended to decide ideal adjuvant treatment: Yes (13.9\% (audience) vs $15.4 \%$ (expert)) and no $(80.6 \%$ (audience) vs $84.6 \%$ (expert)).

B. OncotypeDx testing is recommended for which subset of patients: Node positive, ER/PR positive, HER2neu negative (12.1\% (audience) vs $6.2 \%$ (expert)), node negative, ER/PR positive, HER2neu negative (46.4\% (audience) vs $87.5 \%$ (expert)), node positive, ER/PR negative, HER2neu positive (25.7\% (audience) vs $6.2 \%$ (expert)).

Expert Group Consensus The experts recommended that for molecular profiling of breast cancer, multigene signature testing (Fig. 5) is not the right approach to decide the ideal adjuvant treatment and oncotypeDx testing is strongly recommended for node negative, ER/PR positive, and HER2neu negative patients.

\section{Adjuvant Hormonal Therapy}

The panel drafted five questions for this category. The questions and the response of audiences and experts are provided below:

A. What is the ideal duration for adjuvant hormonal therapy in premenopausal ER/PR+ patients: 5 years $(38.6 \%$ (audience) vs $23.1 \%$ (expert)) and 10 years $(60 \%$ (audience) vs $76.9 \%$ (expert)).

B. In premenopausal hormone receptor positive breast cancer patients, ovarian suppression as adjuvant therapy should be considered in addition to tamoxifen or chemotherapy: In all patients $(28.7 \%$ (audience) vs $18.8 \%$ (expert)), only in high-risk patients (51\% (audience) vs $68.8 \%$ (expert)), not recommended in majority $(18.2 \%$ (audience) vs $12.5 \%$ (expert)).

C. Can some post-menopausal breast cancer patients be adequately treated with tamoxifen alone?: Yes $(69.4 \%$ (audience) vs $57.1 \%$ (expert)) and no $(27.8 \%$ (audience) vs $35.7 \%$ (expert)).

D. In a post-menopausal breast cancer patient, if an AI is used, should it be started up-front: No (7.2\% (audience) vs $16.7 \%$ (expert)), yes-in all patients $(53.6 \%$ (audience) vs $75 \%$ (expert)), and yes-in patients at higher risk (31.9\% (audience) vs $8.3 \%$ (expert)).

E. In post-menopausal breast cancer patients treated with upfront AI, it should be used for 5 years (62.2\% (audience) vs $75 \%$ (expert)), 10 years $(31.9 \%$ (audience) vs $12.5 \%$ (expert)).

Expert Group Consensus For the use of adjuvant hormone therapy, the experts recommended that ideal duration for adjuvant hormonal therapy in premenopausal ER/PR+ patients is 10 years (Fig. 6) and in high-risk patients, ovarian suppression as adjuvant therapy should be considered in addition to tamoxifen or chemotherapy. Some post-menopausal breast cancer patients may or may not be adequately treated with tamoxifen alone. AI in post-menopausal breast cancer patients should be started up-front in all patients and should be used for 5 years. Tamoxifen is found effective in premenopausal and postmenopausal women with hormone-sensitive (ER-positive) breast cancer [25]. Although tamoxifen's use is associated with post-menopausal symptoms such as hot flashes and vaginal discharge [26], its overall risk-benefit ratio is considered favorable for patients, and it should be offered as adjuvant treatment to women with hormone-sensitive breast cancer [27].

\section{Breast Cancer in Young Patients}

The panel drafted two questions for this category. The questions and the response of audiences and experts are provided below:

A. Testing for BRCA1 and -2 mutations is indicated in women $<40$ years: Yes $(65.2 \%$ (audience) vs $75 \%$ (expert)) and no (31.9\% (audience) vs $25 \%$ (expert)).

B. Fertility preservation (e.g., by ovarian tissue or oocyte conservation) should be offered to women $<40$ years: 
Fig. 5 Multigene signature testing

\section{Multigene Signature Testing (Expert Opinion)}

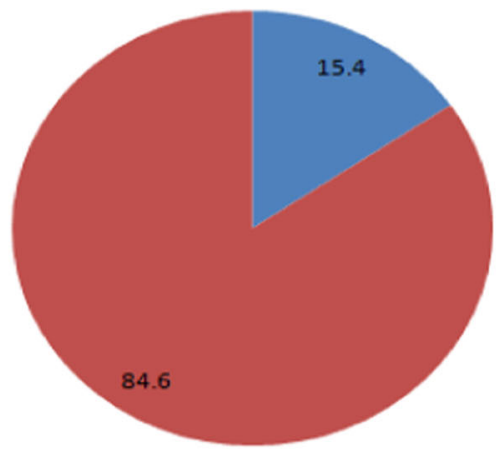

wes

No
Yes $(91.6 \%$ (audience) vs $84.6 \%$ (expert)) and no (4.9\% (audience) vs $7.7 \%$ (expert)).

Expert Group Consensus For breast cancer in young patients, it is recommended to test the BRCA1 and -2 mutations in women $<40$ years of age, and it is highly recommended to offer the fertility preservation to women $<40$ years of age. The patients under 40 years of age comprise about $5 \%$ of the overall breast cancer population, so it's very important to diagnose the disease at early stage [28] and an improved survival with early detection is a valid argument for careful screening among young patients [29].

\section{Conclusion}

The breast cancer expert group had the specific mandate to develop practical consensus recommendations for easy application by the community oncologist. The expert panel considered the existing evidences, current practices in India, and international data and recommended the consensus guidelines which are the perfect blend of the evidences, clinical expertise, and real-life preferences. The consensus guidelines emphasize the need of mandatory core biopsy, metastatic work-up for stage III breast cancer, triple assessment, and clinical breast examination for diagnosis and screening of breast cancer in India.

The guidelines highlighted the recommendations for surgical treatment of $\mathrm{EBC}, \mathrm{LABC}$, management of LABC, use of NACT, and management of axilla in EBC and LABC. Oncoplastic surgical principles were recommended for every breast cancer surgeon. Other key highlights of the recommendations include immediate post-mastectomy breast reconstruction, prophylactic bilateral mastectomy, and prophylactic bilateral salpingo-oophorectomy for risk reduction in BRCApositive women as one of the option, ideal scenarios for use of adjuvant radiotherapy and adjuvant hormone therapy,
Fig. 6 Duration for adjuvant hormonal therapy

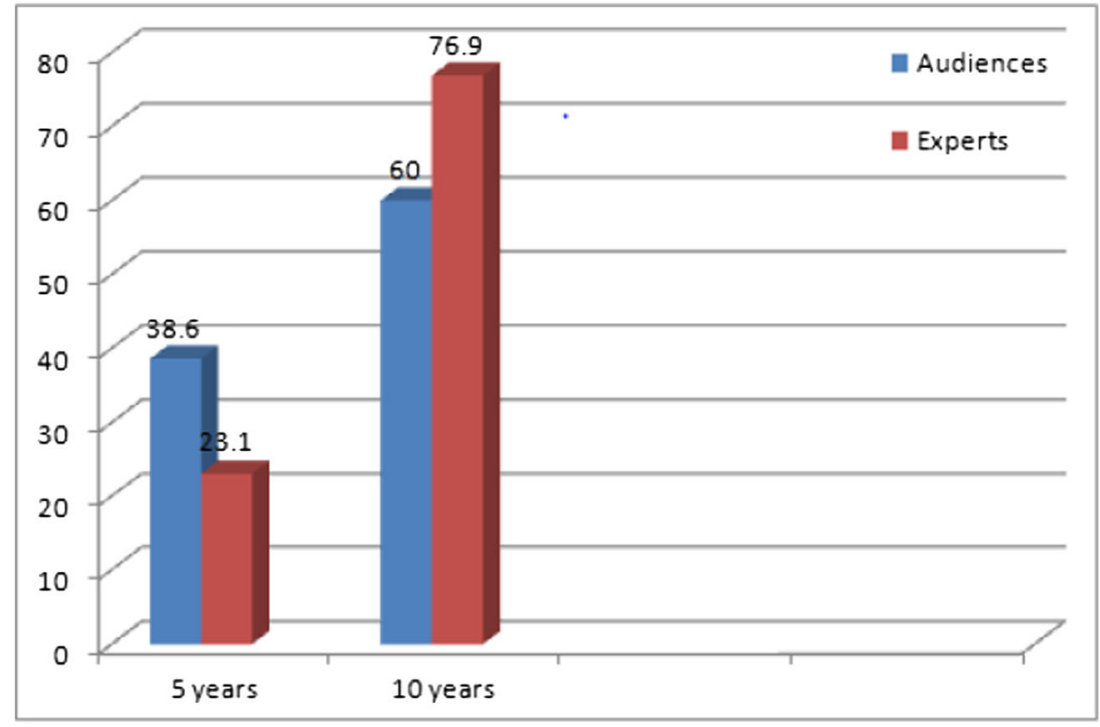


importance of reporting breast histology, molecular profiling of breast cancer, and testing of breast cancer in young patients.

Unresolved issues of importance will be addressed in the updated version of this document as more data becomes available, and the group makes insightful revisions. Therefore, the group encourages gathering real world evidences and optimum treatment options suitable for Indian patients. Although the guidelines must be very useful to the oncology surgeons to utilize these as the best practices, the main challenge will be to focus on its effective implementation and spreading the awareness among the Indian patients. The aim of these consensus guidelines is to find and define the Indian solutions for Indian problems. All those interested in contributing are requested to contact us via email. The aim is to find Indian solutions for Indian problems.

\section{Recommendation Summary}

- For the screening of breast cancer, mandatory core biopsies, metastatic work-up for stage III breast cancer, and triple assessment are recommended.

- The panel emphasizes the need of breast cancer examination to screen the cancer.

- For surgical treatment, the panel recommended no ink on invasive tumor as the minimum acceptable surgical margin and width of margin that needs to be excised is independent of tumor biology; it should not be greater after neo-adjuvant therapy.

- The panel batted for neo-adjuvant chemotherapy as the standard of care for most of the LABC patients.

- The panel recommended that only a subset of LABC can be offered NACT BCS depending on the specifications.

- For the management of axilla in EBC, the experts strongly recommended USG-guided FNAC for preoperative staging of axilla before SLNB/ALND and SLNB should be performed after NACT for the patients planned to be treated with NACT.

- The oncoplastic procedure should be considered if the volume loss is $20 \%$ after BCS and post-mastectomy breast reconstruction should be immediately performed.

- Prophylactic bilateral mastectomy and prophylactic bilateral salpingo-oophorectomy can be performed for risk reduction in BRCA-positive women.

- The experts panel batted for tumor bed radiation boost following BCS for invasive ductal cancer based on selected patients and strongly recommended that APBI should be offered to a select group of early breast cancer patients and for patients with T1 tumor and 1-2 metastatic SLNs.

- The experts advised to omit the radiotherapy for elderly and low-risk patients undergoing BCS.

- The experts strongly recommended that reporting of breast histology must be mandatory in practice.
- For the use of adjuvant hormone therapy in high-risk patients, ovarian suppression as adjuvant therapy should be considered in addition to tamoxifen or chemotherapy.

- The panel strongly recommended testing the BRCA1 and -2 mutations in women $<40$ years of age.

Acknowledgements We would like to thank all expert members involved in the consensus meeting of ABSI and who participated effectively to express their views and depth of practical excellence which enabled us to generate these consensus guidelines and panel of expert scientific team Gaurav Agarval, SVS Deo, and Vani Parmar coordinated with ABSI in identifying relevant questions to be voted and discussed.

\section{References}

1. Ferlay J, Soerjomataram I, Ervik M, Dikshit R, Eser S, Mathers C, et al (2012) Estimated cancer incidence, mortality and prevalance worldwide in 2012..v1.0 (IARC CancerBase No. 11)

2. Global Burden of Disease Cancer Collaboration (2013) The globalburden of cancer. JAMA Oncol 2015

3. Jemal A, Bray F, Melissa MC, Jacques F, Elizabeth W, Forman D (2011) Global cancer statistics. CA Cancer J Clin 61:69-90

4. National Cancer Registry Programme (2013) National Centre for Disease Informatics and Research and Indian Council of Medical Research, three year report of population based cancer registries 2009-2011 National Cancer Registry programme. National Cancer Registry

5. Sharma K, Costas A, Shulman LN, Meara JG (2012) A systematic review of barriers to breast cancer care in developing countries resulting in delayed patient presentation. J Oncol 2012:8

6. Jones S, Johnson K (2012) Women's awareness of cancer symptoms: a review of the literature. Women's Health 8(5):579-591

7. Jones C, Maben J, Jack RH, Davies EA, Forbes LJL, Lucas GR, et al (2014) A systematic review of barriers to early presentation and diagnosis with breast cancer among black women. BMJ Open;4(2).

8. Dey S (2014) Preventing breast cancer in LMICs via screening and/ or early detection: the real and the surreal. World J Clin Oncol 5(3): 509-519

9. Pati S, Hussain MA, Chauhan AS, Mallick D, Nayak S (2013) Patient navigation pathway and barriers to treatment seeking in cancer in India: a qualitative inquiry. Cancer Epidemiol 37(6): 973-978

10. Torre L, Bray F, Siegel RL, Ferlay J, Lortet-Tieulent J, Jemal A (2015) Global cancer statistics, 2012. CA Cancer J Clin 65(2): $87-108$

11. National Breast Cancer Foundation, INC. Available from http:// www.nationalbreastcancer.org/breast-cancer-treatment

12. Breast Cancer Deadline 2020 (2012). Retrieved May 10, 2014, from www.breastcancerdeadline2020.org/

13. Harbeck N, Thomssen C, Gnant M (2013) St. Gallen 2013: brief preliminary summary of the consensus discussion. Breast Care 8 : 102-109

14. Gnant M, Thomssen C, Harbeck N (2015) St. Gallen/Vienna 2015: a brief summary of the consensus discussion. Breast Care 10:124 130

15. Chauhan A, Sharma MM (2016) Evaluation of surgical outcomes following oncoplastic breast surgery in early breast cancer and comparison with conventional breast conservation surgery. Med J Armed Forces India 72(1):12-18 
16. Tryfonidis K, Senkus E, Cardoso MJ et al (2015) Management of locally advanced breast cancer-perspectives and future directions. Nat Rev Clin Oncol 12(3):147-162

17. Vijaykumar DK, Arunlal M (2015) Management of axilla in 2015 in Indian scenario. Indian J Surg Oncol 6(4):435-439

18. Rolph R, Mehta S, Farhadi J (2016) Breast reconstruction: options post-mastectomy. Br J Hosp Med (Lond) 77(6):334-342

19. Guillem JG, Wood WC, Moley JF et al (2006) ASCO/SSO review of current role of risk-reducing surgery in common hereditary cancer syndromes. J Clin Oncol 24(28):4642-4660

20. Hartmann LC, Schaid DJ, Woods JE et al (1999) Efficacy of bilateral prophylactic mastectomy in women with a family history of breast cancer. N Engl J Med 340(2):77-84

21. Domchek SM, Friebel TM, Singer CF et al (2010) Association of risk-reducing surgery in BRCA1 or BRCA2 mutation carriers with cancer risk and mortality. JAMA 304(9):967-975

22. Rebbeck TR, Friebel T, Lynch HT et al (2004) Bilateral prophylactic mastectomy reduces breast cancer risk in BRCA1 and BRCA2 mutation carriers: the PROSE Study Group. J Clin Oncol 22(6): $1055-1062$
23. Meijers-Heijboer H, van Geel B, van Putten WL et al (2001) Breast cancer after prophylactic bilateral mastectomy in women with a BRCA1 or BRCA2 mutation. N Engl J Med 345(3):159-164

24. Peto R, Boreham J, Clarke M et al (2000) UK and USA breast cancer deaths down $25 \%$ in year 2000 at ages 20-69 years [letter]. Lancet 355:1822

25. Early Breast Cancer Trialists' Collaborative Group (1998) Tamoxifen for early breast cancer: an overview of the randomized trials. Lancet 351:1451-1467

26. Fisher B, Costantino JP, Wickerham DL et al (1998) Tamoxifen for prevention of breast cancer: report of the National Surgical Adjuvant Breast and Bowel Project P-1 Study. J Natl Cancer Inst 90:1371-1388

27. Chew HK (2001) Adjuvant therapy for breast cancer: who should get what? West J Med 174(4):284-287

28. Sariego J (2010) Breast cancer in the young patient. Am Surg 76(12):1397-1400

29. Sariego J, Zrada S, Byrd M et al (1995) Breast cancer in young patients. Am J Surg 170(3):243-245 